\title{
Micropatterning in bistable cholesteric device with Bragg's reflection
}

\author{
M.I. Gritsenko ${ }^{1}$, S.I. Kucheev ${ }^{1}$, P.M. Lytvyn ${ }^{2}$, V.G. Tishenko ${ }^{3}$, V.M. Tkach $^{4}$, V.B. Yelshansky ${ }^{4}$ \\ ${ }^{1}$ Chernigov State University, 53, G. Polubotka str., 14038 Chernigov, Ukraine, e-mail: skucheev@yahoo.com \\ ${ }^{2} V$. Lashkaryov Institute of Semiconductor Physics, 45, prospect Nauky, 03028 Kyiv, Ukraine \\ ${ }^{3}$ Kharkov State University, Kharkov, Ukraine \\ ${ }^{4}$ Institute of Super-Hard Materials, Kyiv, Ukraine
}

\begin{abstract}
In this work, the method to form bistable patterning in a cholesteric cell with Bragg's reflection in the visible spectral range is demonstrated. In order to reach this, we used a $\mathrm{Si} / \mathrm{SiO}_{2}$ structure in which a potential relief on $\mathrm{SiO}_{2}$ surface is induced due to enrichment and depletion of required form areas of the silicon near-surface layer during external electric field action. The patterning of enrichment is determined by the positive charge embedded into $\mathrm{SiO}_{2}$ film, which is formed by thermodiffusion of aluminum atoms.
\end{abstract}

Keywords: liquid crystal, selective reflection, silicon, patterning electrode.

Manuscript received 21.12.05; accepted for publication 29.03.06.

\section{Introduction}

Cholesteric liquid crystals (CLCs) possessing Bragg's reflection in the visible spectral range are utilized in a variety of polarizerless bistable displays $[1,2]$. There are also a good perspective of application of Bragg's cholesterics in electrically driven diffraction gratings, dye doped LC lasing with periodic patterning. In the latter case, a flat phase-locked laser can be developed.

In order to realize unique properties of Bragg's cholesterics in applications, it is necessary to develop an effective micropatterning of the LC layer. Such methods of surface treatments as photoalignment [3, 4], ion bombardment [5], AFM scanning microscopy technique [6], tilted plasma [7], self-assembled gels and networks [8] give good results for alignment and patterning the nematics or cholesterics having large helix pitch but absolutely unsuitable for patterning the Bragg cholesteric with reflection in the visible spectral range.

In currently Bragg devices, a conventional indiumtin oxide (ITO) electrode patterning is used. However, ITO patterning has some disadvantages associated with the appearing relief. The ITO surface relief can play a role of a source of light wave diffraction that impairs particularly a performance of diffraction grating, LC wave guides, lasing in LCs.

It is well known $[9,10]$ that $\mathrm{Si} / \mathrm{SiO}_{2}$ structures are often used as a photosensitive part of liquid crystal spatial light modulators (LCSLM). A photosensitive state of $\mathrm{Si} / \mathrm{SiO}_{2}$ structure is achieved using the external electric field due to the so-called field effect. In this state, there is a depletion of major carriers within the near-surface layer of silicon, and, accordingly, the electric resistivity of this layer increases. The $\mathrm{Si} / \mathrm{SiO}_{2}$ structure transforms the written light distribution to the potential relief on the $\mathrm{SiO}_{2}$ surface owing to nonequilibrium carrier generation within the depleted layer. In accordance with this potential relief a LC director reorients and modulates reading light in LCSLM.

$\mathrm{SiO}_{2}$ dielectric film in the $\mathrm{Si} / \mathrm{SiO}_{2}$ structure performs at least two functions. First, the $\mathrm{SiO}_{2}$ film prevents charge carriers to transfer from LC into silicon that enables to form a depleted layer. Second, this $\mathrm{SiO}_{2}$ film plays a role of a dielectric mirror which reflects reading light in LCSLM.

This work shows that the issue of reliefless patterning for a cholesteric cell with Bragg's reflection can be solved by means of using the $\mathrm{Si} / \mathrm{SiO}_{2}$ structure with an embedded quasi-electrode within the $\mathrm{SiO}_{2}$ film. The ion charge formed in the $\mathrm{SiO}_{2}$ film due to thermodiffusion of aluminum atoms plays a role of a gate that permanently enriches the silicon near-surface layer. During control pulse action, the patterned quasielectrode prevents depletion of the silicon near-surface layer, whereas in the rest part of silicon there observed an electron depletion.

\section{Experiment}

In our experiment, as a $\mathrm{Si} / \mathrm{SiO}_{2}$ structure we used a silicon substrate of the $n$-type with the specific resistance 4.5 Ohm.cm containing $p^{+}$-pockets. On the silicon surface, the dielectric amorphous $\mathrm{SiO}_{2}$ film was thermally grown with the thickness of $0.4 \mu \mathrm{m}$. 
An embedded quasi-electrode formation and its micropatterning within $\mathrm{SiO}_{2}$ film were made in the following way. On the silicon oxide film, the aluminum film of about $0.7-\mu \mathrm{m}$ thickness was thermally deposited. Using photolitographic and selective etching processes, the aluminum film was patterned on the $\mathrm{SiO}_{2}$ surface as shown in Fig. 1a. Usually, the thermal annealing of the aluminum film on silicon oxide results in appearance of alumino-silicate nanoscale islands on the $\mathrm{SiO}_{2}$ surface or its change at the nanoscale level $[11,12]$. In order to avoid any surface changes at the nanolevel, the $\mathrm{Si} / \mathrm{SiO}_{2}$ structure was annealed at the temperature lower than that necessary to obtain a good adhesion of aluminum to silicon oxide [13]. After annealing, the patterned aluminum film was removed by selective etching (Fig. 1b).

The investigation of the $\mathrm{SiO}_{2}$ surface by using an optical microscope showed that "A" and "B" type of surfaces are optically identical (Fig. 1b).

Then, the $\mathrm{SiO}_{2}$ surface was investigated using a scanning electron microscope (SEM). It was found that "A" areas look like darker than "B" areas (Fig. 2, "A" and "B" vertical areas). There takes place such darkening over both the $n$-type and $p^{+}$-type conductivity silicon substrate surface (Fig. 2). "A" area darkening in comparison with "B" one means that the electron beam of SEM penetrates into the oxide film at a certain depth ( $\sim 50 \mathrm{~nm}$ [14]) and additionally scatters by the defect layer which appears because of the aluminum ions are located here. So, due to thermodiffusion of aluminum atoms a static positive charge $\mathrm{Q}^{+}$is formed in the $\mathrm{SiO}_{2}$ film, Fig. 1b. The shape and size of charge location exactly repeats the patterning of annealed aluminum film. Just under this positive charge there is an electron enrichment $\mathrm{Q}^{-}$, Fig. $1 \mathrm{~b}$.

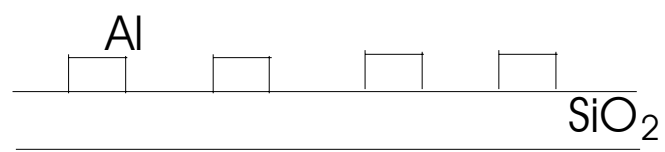

$\mathrm{Si}$

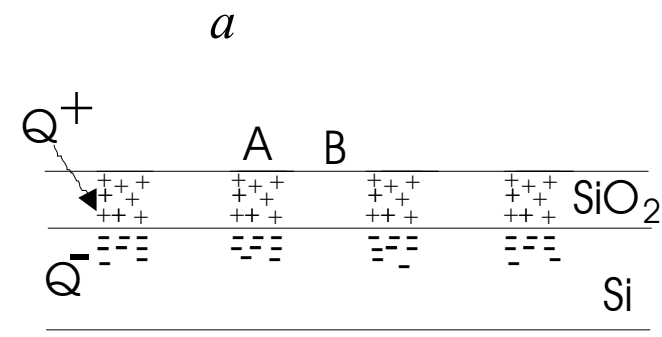

$b$

Fig. 1. Schematic representation of preparing the charge embedded into the $\mathrm{Si} / \mathrm{SiO}_{2}$ structure. a) Patterning the aluminum film on the $\mathrm{SiO}_{2}$ surface. b) Formation of the embedded charge $\mathrm{Q}^{+}$(quasi-electrode) after annealing and selective wet etching of the aluminum film. $\mathrm{Q}^{-}-$permanent electron enrichment. " $\mathrm{A}$ " and "B" types of surface are those from which aluminum film was removed after and before annealing, respectively. $p^{+}$-pockets are not shown.

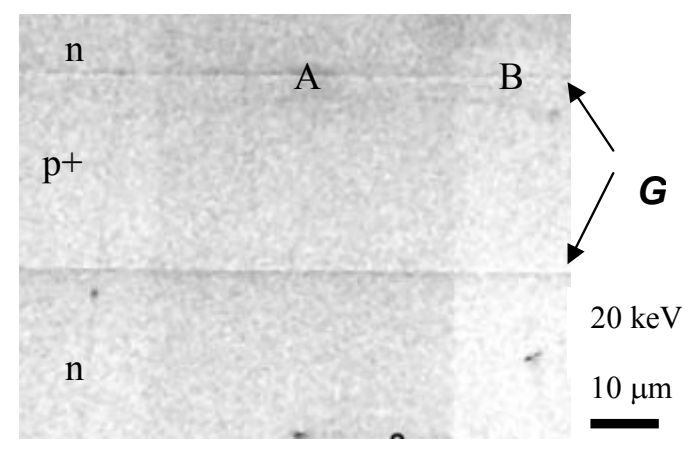

Fig. 2. SEM micrograph of $\mathrm{SiO}_{2}$ surface on the $n$-type silicon substrate with $p^{+}$-pocket after annealing and selective wet removing of aluminum pattern. Dark vertical area " $A$ " is a track of the annealed aluminum film. "B" area is a surface from which the aluminum film was removed before annealing. "G" is the boundary between $p^{+}$-and $n$-type conductivity areas.

The "A" and "B" surfaces were examined using an atomic force microscope (AFM, Digital Instruments NanoScope IIIa in the tapping mode) in the scanned areas of $1 \times 1 \mu \mathrm{m}$. The surface topography in the points "A" and "B" as the height in 2D maps and their corresponding reliefs are shown in Fig. 3a, b. As it is well seen, the morphology of these fragments of " $A$ " and "B" surfaces are almost identical. As seen, relief deference between these " $A$ " and " $B$ " surfaces is less than $1.5 \ldots 2 \mathrm{~nm}$. This means that such a deference between the "A" and "B" relief cannot be a source of light wave diffraction in the visible range.

The LC cell was assembled using a $\mathrm{Si} / \mathrm{SiO}_{2}$ substrate treated in the above manner and an upper reference ITO substrate (1), which is coated with a rubbed polyimide film (2), as schematically shown in Fig. 4a. The cell was filled with the chiral (Fig. 4b) doped nematic (LC1268) with equilibrium helix equal $\sim 400 \mathrm{~nm}$ having selective
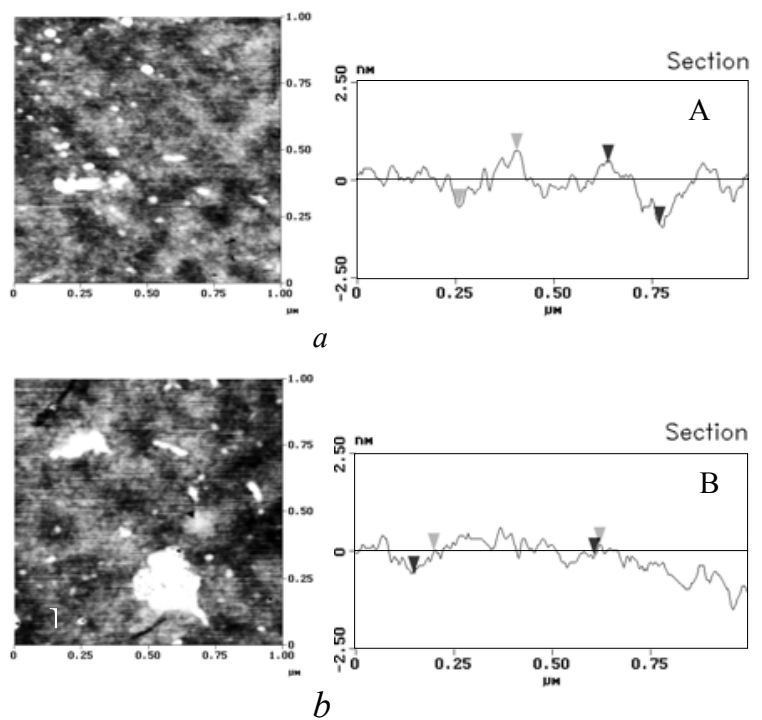

Fig. 3. 2D AFM images of "A"(a) and "B"(b) surfaces and their typical reliefs, respectively. 
reflection at $625 \mathrm{~nm}$ in visible. Thickness of cholesteric layer was approximately equal to $1 \mu \mathrm{m}$.

Recording a pattern in such a cell with the cholesteric layer is carried out as follows. Simultaneously, a direct voltage with $+U$-polarity on silicon and alternating one are applied to the cell. The DC voltage bias causes an electron depletion of silicon near-surface. Therefore, the depletion layer boundary $(L)$ lowers (Fig. 4a) and the resistivity of the depleted layer increases. Here, a certain part of the external voltage drops on the depleted layer. On the other hand, just under the quasi-electrode, a silicon surface is not depleted because there is an initial electron enrichment (EE), Fig. 4a. Therefore, the external voltage applied to cell drops entirely on the cholesteric layer. Thus, there a potential relief forms on the $\mathrm{SiO}_{2}$ surface which is identical to the quasi-electrode pattern. The potential relief difference between " $A$ " and " $B$ " areas runs up to several volts that is enough for transition into a focal conic texture over "B" areas and, at the same time, for planar $\rightarrow$ homeotropic transition over "A" areas, Fig. 5a. Fig. $5 \mathrm{~b}$ confirms that the silicon surface between quasielectrodes is depleted and photosensitive. As well seen, the laser spot on "B" area causes a confocal $\rightarrow$ homeotropic transition. This means that under the action of light irradiation the non-equilibrium electronhole pairs are generated, and the near-surface silicon resistivity decreases here.

After switching off of the voltage, the homeotropic texture over " $A$ " areas relaxes to the planar texture with Bragg's reflection while the cholesteric one over " $\mathrm{B}$ " area remains in the focal conic scattering texture (Fig. 6a). In order to erase the pattern, a high voltage has to be applied to switch a cholesteric texture over " $\mathrm{B}$ " areas to the homeotropic one. Then the cell completely relaxes back to the planar texture, Fig. $6 \mathrm{~b}$.

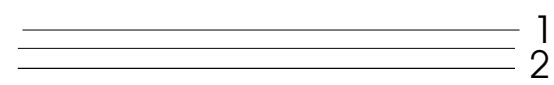

3
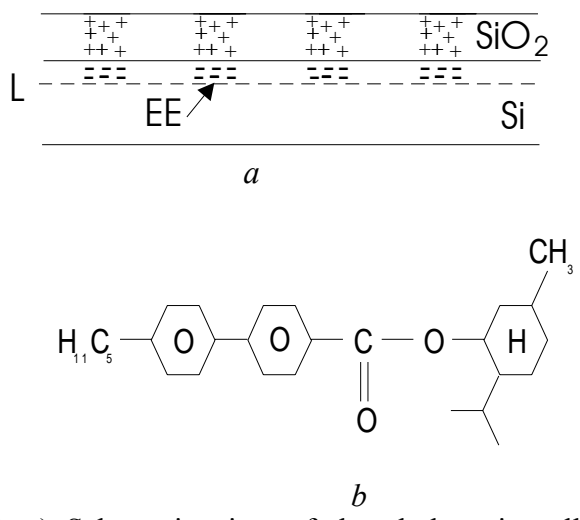

Fig. 4. a) Schematic view of the cholesteric cell based on the $\mathrm{Si} / \mathrm{SiO}_{2}$ structure. 1 - ITO electrode, 2 - rubbed polyimide layer for alignment of cholesteric, 3 - cholesteric layer, L near-surface depletion boundary, EE - electron enrichment. b) Chiral component of cholesteric-nematic mixture.

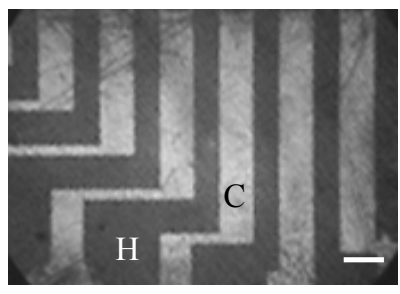

$a$

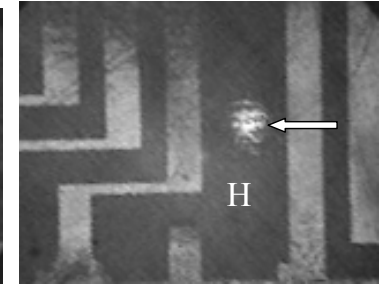

$b$
Fig. 5. a) Homeotropic (H) texture over quasi-electrodes ("A" areas) and focal conic (C) one over depleted areas of silicon ("B" areas). Frequency and AC voltage were $10^{5} \mathrm{~Hz}$ and $45 \mathrm{~B}$, respectively. The DC voltage bias was $+3 \mathrm{~V}$. The bar is $50 \mu \mathrm{m}$. b) Focal conic $\rightarrow$ homeotropic transition under $\mathrm{He}-\mathrm{Ne}$ laser illumination. The arrow points on the laser spot.

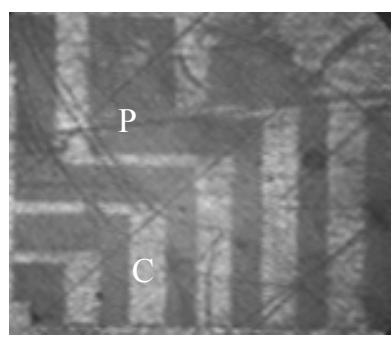

$a$

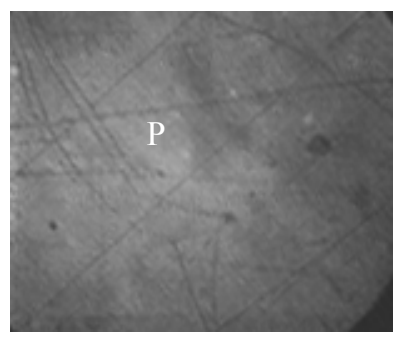

$b$
Fig. 6. a) Written patterning in a cholesteric layer. $\mathrm{P}$ - planar texture with Bragg's reflection, $\mathrm{C}$ - focal conic scattering texture. b) Erased pattern after high voltage pulse action.

It should be noted that the pattern is not associated with any surface contamination or a possible rest of aluminum film after etching. If the $\mathrm{SiO}_{2}$ surface is etched in the layer-by-layer manner, the above described pattern can be recorded after each stage of etching and assembling of the cell. This confirms that the effect of patterning is associated with positive aluminum ion penetration and distribution inside $\mathrm{SiO}_{2}$ from its surface to the silicon one during annealing.

\section{Conclusion}

Thermal diffusion of aluminum atoms from the aluminum pattern film causes the formation of the positive charge embedded into $\mathrm{SiO}_{2}$ (quasi-electrode). This embedded charge faithfully copies the pattern of the aluminum film and permanently enriches the silicon near-surface layer in the $\mathrm{Si} / \mathrm{SiO}_{2}$ structure. Such $\mathrm{Si} / \mathrm{SiO}_{2}$ structures are used as a substrate in a cholesteric cell with Bragg's reflection. The potential relief on the $\mathrm{SiO}_{2}$ surface is achieved when applying a pulse of the driving voltage to a cell. The "relief" amplitude is enough for simultaneous transitions of planar $\rightarrow$ focal conic textures over depleted silicon and planar $\rightarrow$ homeotropic ones over quasi-electrode, respectively. This enables to obtain the rewritten patterned areas with Bragg's reflection in a cholesteric cell. 
References

1. S.T. Wu, D.K. Yang, Reflective liquid crystal displays. John Willey\& Sons, Ltd (2001).

2. A. Kozachenko, P.Oleksenko, V. Sorokin, V.Nazarenko // IDRC'97, p.148-151 (1997).

3. M.Schadt, H. Seiberle, A.Schuster // Nature, 381, 212(1996).

4. M. Schadt, K. Schmit, V. Kozinkov, V. Chigrinov // Jpn J. Appl. Phys. 31, p. 2155 (1992).

5. Z.M. Sun, J.M. Engels, I. Dozov and G. Durand // J. De Phys. 2, p. 59-73 (1994).

6. B. Wen, M.P. Mahajan, C. Rosenblatt // Appl. Phys. Lett. 76, No 10, p. 1240-1242 (2000)

7. O. Yaroshchuk, Yu. Zakrevskyy, A. Dobrovolsky, S. Pavlov // Proc. SPIE, 4418, p. 49 (2001).

8. Y. Zhao, X.Tong // Adv. Mater. 15, No 17, p. 14311435 (2003).
9. A.A. Vasiliev et al. // Optic, 67(3), p. 223-236 (1984).

10. N.F. Kovtonyuk, E.N. Sal'nikov, Photosensitive MOS-devices for image transducers. Radio i svyaz', Moscow (1990) (in Russian).

11. M.I. Gritsenko, S.I. Kucheev, P.M. Litvin // Proc. of 13th SID Symposium"Advanced Display Technologies", Raubichi (Belarus), September 7-10, p. $207-$ 210, 2004.

12. N.I. Gritsenko, S.I. Kucheev, P.M. Litvin, Nematic diffractive grating induced by nanotreated silicon oxide surface // Proc. of 11th IDW'04, Japan, 2004.

13. R.M. Burger, R.P. Donovan, Oxidation, diffusion and epitaxy. Fundamentals of silicon integrated device technology. Vol.1. Mir, Moscow (1969) (in Russian).

14. S.G. Konnikov, A.F. Sidorov, Electronic probe methods of semiconductor materials and devices investigation. Energiya, Moscow (1978) p.134 (in Russian). 